\title{
Neurophysiological Evidence for a Cortical Contribution to the Wakefulness-Related Drive to Breathe Explaining Hypocapnia-Resistant Ventilation in Humans
}

\author{
- Matthieu Dubois, ${ }^{1,2 *}$ Cécile Chenivesse, ${ }^{1,2 *}$ Mathieu Raux, ${ }^{1,3}$ Adrian Morales-Robles, ${ }^{1}$ Marie-Cécile Nierat, ${ }^{1}$ \\ @Gilles Garcia, ${ }^{1}$ @Xavier Navarro-Sune, ${ }^{1,4}$ Mario Chavez, ${ }^{4}$ @Jacques Martinerie, ${ }^{4}$ and ${ }^{-}$Thomas Similowski ${ }^{1,2}$ \\ ${ }^{1}$ Sorbonne Université, Université Paris 06 Pierre et Marie Curie and Institut National de la Santé et de la Rercherche Médicale, Unité Mixte de Recherche- \\ Santé 1158 Neurophysiologie Respiratoire Expérimentale et Clinique, F-75013 Paris, France, ${ }^{2}$ Assistance Publique-Hôpitaux de Paris, Groupe Hospitalier \\ Pitié-Salpêtrière Charles Foix, Service de Pneumologie et Réanimation Médicale Département R3S, F-75013 Paris, France, ${ }^{3}$ Assistance Publique-Hôpitaux \\ de Paris, Groupe Hospitalier Pitié-Salpêtrière Charles Foix, Département d’Anesthésie-Réanimation, F-75013 Paris, France, and ${ }^{4}$ Centre National de la \\ Recherche Scientifique, Unité Mixte de Recherche 7225, Institut du Cerveau et de la Moëlle, F-75013 Paris, France
}

Spontaneous ventilation in mammals is driven by automatic brainstem networks that generate the respiratory rhythm and increase ventilation in the presence of increased carbon dioxide production. Hypocapnia decreases the drive to breathe and induces apnea. In humans, this occurs during sleep but not during wakefulness. We hypothesized that hypocapnic breathing would be associated with respiratory-related cortical activity similar to that observed during volitional breathing, inspiratory constraints, or in patients with defective automatic breathing (preinspiratory potentials). Nineteen healthy subjects were studied under passive (mechanical ventilation, $n=10$ ) or active (voluntary hyperventilation, $n=9$ ) profound hypocapnia. Ventilatory and electroencephalographic recordings were performed during voluntary sniff maneuvers, normocapnic breathing, hypocapnia, and after return to normocapnia. EEG recordings were analyzed with respect to the ventilatory flow signal to detect preinspiratory potentials in frontocentral electrodes and to construct time-frequency maps. After passive hyperventilation, hypocapnia was associated with apnea in 3 cases and ventilation persisted in 7 cases ( 3 and 6 after active hyperventilation, respectively). No respiratoryrelated EEG activity was observed in subjects with hypocapnia-related apneas. In contrast, preinspiratory potentials were present at vertex recording sites in 12 of the remaining 13 subjects $(p<0.001)$. This was corroborated by time-frequency maps. This study provides direct evidence of a cortical substrate to hypocapnic breathing in awake humans and fuels the notion of corticosubcortical cooperation to preserve human ventilation in a variety of situations. Of note, maintaining ventilatory activity at low carbon dioxide levels is among the prerequisites to speech production insofar as speech often induces hypocapnia.

Key words: cerebral cortex; control of breathing; hypocapnia; respiration

\section{Significance Statement}

Human ventilatory activity persists, during wakefulness, even when hypocapnia makes it unnecessary. This peculiarity of human breathing control is important to speech and speech-breathing insofar as speech induces hypocapnia. This study evidences a specific respiratory-related cortical activity. This suggests that human hypocapnic breathing is driven, at least in part, by cortical mechanisms similar to those involved in volitional breathing, in breathing against mechanical constraints or with weak inspiratory muscle, and in patients with defective medullary breathing pattern generators. This fuels the notion that the human ventilatory drive during wakefulness often results from a corticosubcortical cooperation, and opens new avenues to study certain ventilatory and speech disorders.

\section{Introduction}

Spontaneous ventilation in mammals is driven by the automatic activity of brainstem neural networks (Feldman et al., 2013) gener-

Received July 27, 2016; revised Aug. 27, 2016; accepted Aug. 29, 2016.

Author contributions: M.D., C.C., A.M.-R., G.G., J.M., and T.S. designed research; M.D., C.C., A.M.-R., M.-C.N., G.G., and T.S. performed research; M.D., C.C., M.R., A.M.-R., M.-C.N., G.G., X.N.-S., M.C., J.M., and T.S. analyzed data; M.D., C.C., M.R., A.M.-R., M.-C.N., G.G., X.N.-S., M.C., J.M., and T.S. wrote the paper ating the respiratory rhythm and adapting the ventilatory excretion of carbon dioxide $\left(\mathrm{CO}_{2}\right)$ to metabolic needs (chemosensitivity). Consequently, hypercapnia (defined as an increase in $\mathrm{CO}_{2}$ in the arterial blood, $\mathrm{Pa}_{\mathrm{CO} 2}$ ), acidosis, fever, and exercise automatically in- 
crease ventilation. Hypocapnia (low $\mathrm{Pa}_{\mathrm{CO} 2}$ ) on the other hand, depresses the activity of pre-Bötzinger and Bötzinger neurons within respiratory central pattern generators (Sun et al., 2001) with a breakdown of raphe-pontomedullary connectivity (Nuding et al., 2015), and induces apneas. In other words, there is no need to breathe when there is no $\mathrm{CO}_{2}$ to expel. In animals, hypocapnia arrests breathing during sleep and during wakefulness (with a higher apnea threshold). In humans during anesthesia (Hanks et al., 1961) and sleep (Henke et al., 1988; Datta et al., 1991), decreasing the arterial blood partial pressure of $\mathrm{CO}_{2}\left(\mathrm{~Pa}_{\mathrm{CO} 2}\right)$ by a few $\mathrm{mmHg}$ suffices to induce apneas. In contrast, human ventilation resists hypocapnia during wakefulness (so-called wakefulness drive to breathe) (Fink, 1961). This is true after active hyperventilation (Corfield et al., 1995) and after passive hyperventilation administered via a mechanical ventilator (Datta et al., 1991; Corfield et al., 1995). This observation is all the more surprising in that passive hyperventilation not only lowers $\mathrm{Pa}_{\mathrm{CO}}$ but also exerts a nonchemical inhibitory influence on breathing control (Fauroux et al., 1998; Hopkinson et al., 2012). The mechanisms accounting for the resistance of human spontaneous ventilation to hypocapnia have not been clearly identified. Behavioral influences have been called upon (Datta et al., 1991; Corfield et al., 1995; Shea, 1996), and several hypotheses have been proposed (for review, see Shea, 1996). However, no recent data are available on this topic, and none of the proposed hypotheses has been tested experimentally. Yet this is a relevant issue insofar as maintaining ventilatory activity at low carbon dioxide levels is among the prerequisites to speech production insofar as speech often induces hypocapnia (Bunn and Mead, 1971).

In addition to the medullary automatic respiratory circuitry, cortical networks can also drive breathing activities. Voluntary breathing commands can arise from higher brain structures, as respiratory muscles are represented in the primary motor cortex and premotor structures (Smith, 1938; Gandevia and Rothwell, 1987; Macefield and Gandevia, 1991; Similowski et al., 1996; Hudson et al., 2016). Neuronal activities of the premotor cortex and supplementary motor area (SMA) are also involved when humans are exposed to inspiratory constraints, either experimental (Raux et al., 2007; Raux et al., 2013) or disease-related (Launois et al., 2015; Georges et al., 2016) and during speech breathing (Tremoureux et al., 2014b). In patients with deficient respiratory automatism due to a PHOX 2B mutation (congenital central hypoventilation syndrome or Ondine's curse) who must remain awake to breathe, a respiratory-related cortical activity is present during spontaneous breathing, with electroencephalographic signs of SMA activation (slow preinspiratory potentials resembling movement preparation potentials) (Tremoureux et al., 2014a). This finding has been interpreted in terms of corticosubcortical cooperation to produce the wakefulness-related neural drive to breathe (Tremoureux et al., 2014a). It has also been demonstrated that the cerebral cortex, and more precisely the SMA, exerts a tonic respiratory facilitatory activity on breathing (Laviolette et al., 2013) because inhibitory conditioning of the SMA by repetitive transcranial magnetic stimulation depresses

d'Etude et de Recherche du Corps Médical and CARDIF- I'Assistance Respiratoire, Fontenay-aux-Roses scholarship (Paris). G.G. was supported by Fonds de Dotation Recherche en Santé Respiratoire-Fondation du Souffle scholarship (Paris).

The authors declare no competing financial interests.

${ }^{*}$ M.D. and C.C. contributed equally to this study as first authors.

Correspondence should be addressed to Dr. Thomas Similowski, Service de Pneumologie et Réanimation Médicale, Groupe Hospitalier Pitié Salpêtrière Charles Foix, 47-83 boulevard de l'Hôpital, 75651 Paris Cedex 13, France. E-mail: thomas.similowski@psl.aphp.fr.

DOI:10.1523/JNEUROSCI.2376-16.2016

Copyright $\odot 2016$ the authors $\quad 0270-6474 / 16 / 3610674-10 \$ 15.00 / 0$ the excitability of the corticospinal pathway to the diaphragm (Laviolette et al., 2013).

The present study was designed to review hypocapnic breathing in the light of recent progress in the understanding of cortical breathing control. We hypothesized that the maintenance of ventilation following deep hypocapnia induced by hyperventilation would be associated with activation of cortical respiratory networks. To test this hypothesis, we recorded EEGs in normal individuals during quiet normocapnic breathing, during sustained passively and actively induced hypocapnia, and after return to baseline $\mathrm{Pa}_{\mathrm{CO} 2}$. EEGs were processed with respect to ventilatory flow, as previously described (Raux et al., 2007), to identify preinspiratory potentials. For corroboratory purposes, EEGs were also processed by using a less specific but more robust timefrequency approach.

\section{Materials and Methods}

Main set of experiments (hypocapnia induced by

passive hyperventilation)

Subjects and ethical approval. Ten healthy subjects (mean age 22 years; range 21-24 years; 5 men, 5 women; mean body mass index $21.8 \mathrm{~kg} / \mathrm{m}^{2}$, range $20.8-24.1 \mathrm{~kg} / \mathrm{m}^{2}$ ) participated in this study. None of them had previously participated in a respiratory physiology or neurophysiology protocol, and none of them performed any forms of activity involving voluntary control of breathing (e.g., yoga, swimming, acting, singing, wind instruments). The subjects had no history of respiratory disease, they were all nonsmokers and did not take any medications. Spirometry, performed in all subjects according to current guidelines, confirmed the absence of any obstructive or restrictive ventilatory disorders. All subjects had a Nijmegen score of $<23$ of 64 , making the presence of a chronic hyperventilation syndrome unlikely (van Dixhoorn and Duivenvoorden, 1985; van Dixhoorn and Folgering, 2015). On the day of the experiment, the subjects were not sleep deprived and had not consumed alcohol or any other psychoactive substances for at least $24 \mathrm{~h}$.

The study had been previously approved by the appropriate regulatory and ethical authority (Comite de Protection des Personnes Ile-de-France 6 La Pitié-Salpêtrière, Paris). All subjects provided their written consent to participate after having been informed about the general study procedures, the methods used, and the absence of risk for their health or any residual effects. The precise objective of the study was only revealed after the study to preserve the naiveté necessary for the interpretation of the results.

Ventilatory signals. Ventilatory flow was recorded by means of a mouthpiece with occlusion of the nose by a pneumotachograph (New Single use Adult/Pediatric Flow Sensor PN 281637, Hamilton Medical ) connected to a differential pressure transducer (Spirometer module, AD Instruments; RRID:SCR_001620). Expired gas was collected at the mouth and transferred to an infrared gas analyzer (Servomex, La Plaine Saint-Denis) to determine the expired fraction of carbon dioxide and to calculate the end-tidal carbon dioxide pressure $\left(\mathrm{Pet}_{\mathrm{CO} 2}\right)$. Ventilatory flow and $\mathrm{Pet}_{\mathrm{CO} 2}$ signals were digitized at a sampling frequency of $200 \mathrm{~Hz}$ and were recorded for subsequent analysis (PowerLab and LabChart 7.2 for PC, AD Instruments; RRID:SCR_001620).

Electroencephalographic signals. The electroencephalographic signal was recorded by means of 12 equidistant scalp electrodes (ActiCap, BrainProducts), according to the conventional 10-20 topographic system (Rektor, 2002) at locations Fp1, Fp2, Fz, F3, F4, FCZ, Cz, C3, C4, Pz, $\mathrm{P} 3, \mathrm{P} 4$, on the earlobes at A1 and A2 with the earth at AFZ (ActiCAP, BrainProducts). The EEG signal was amplified, digitized at a sampling frequency of $2000 \mathrm{~Hz}$, and recorded for subsequent analysis (V-Amp and Recorder software, BrainProducts).

Experimental conditions and protocol. During the experimental sessions, subjects were comfortably seated in an armchair with the head and neck maintained by a cushion, in a warm room in semidarkness. Their attention was captured by watching a film. Subjects were instructed to remain calm and relaxed without falling asleep and to avoid moving 


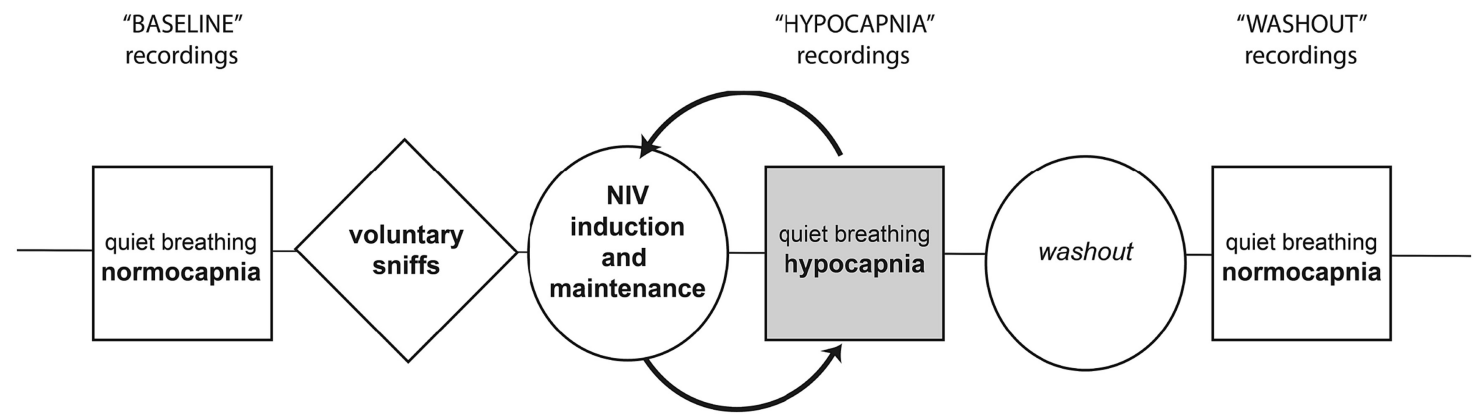

Figure 1. Flow chart of the experimental protocol. All recordings were performed during calm normocapnic breathing (baseline recordings), during rapid voluntary inspirations (sniffs), then after induction of hypocapnia by passive hyperventilation ( $\mathrm{Pet}_{\mathrm{CO} 2} 20-30 \mathrm{mmHg}$ ) (hypocapnia recordings), and finally after return to $\mathrm{Pet}_{\mathrm{CO} 2}>35 \mathrm{mmHg}$ (washout recordings).

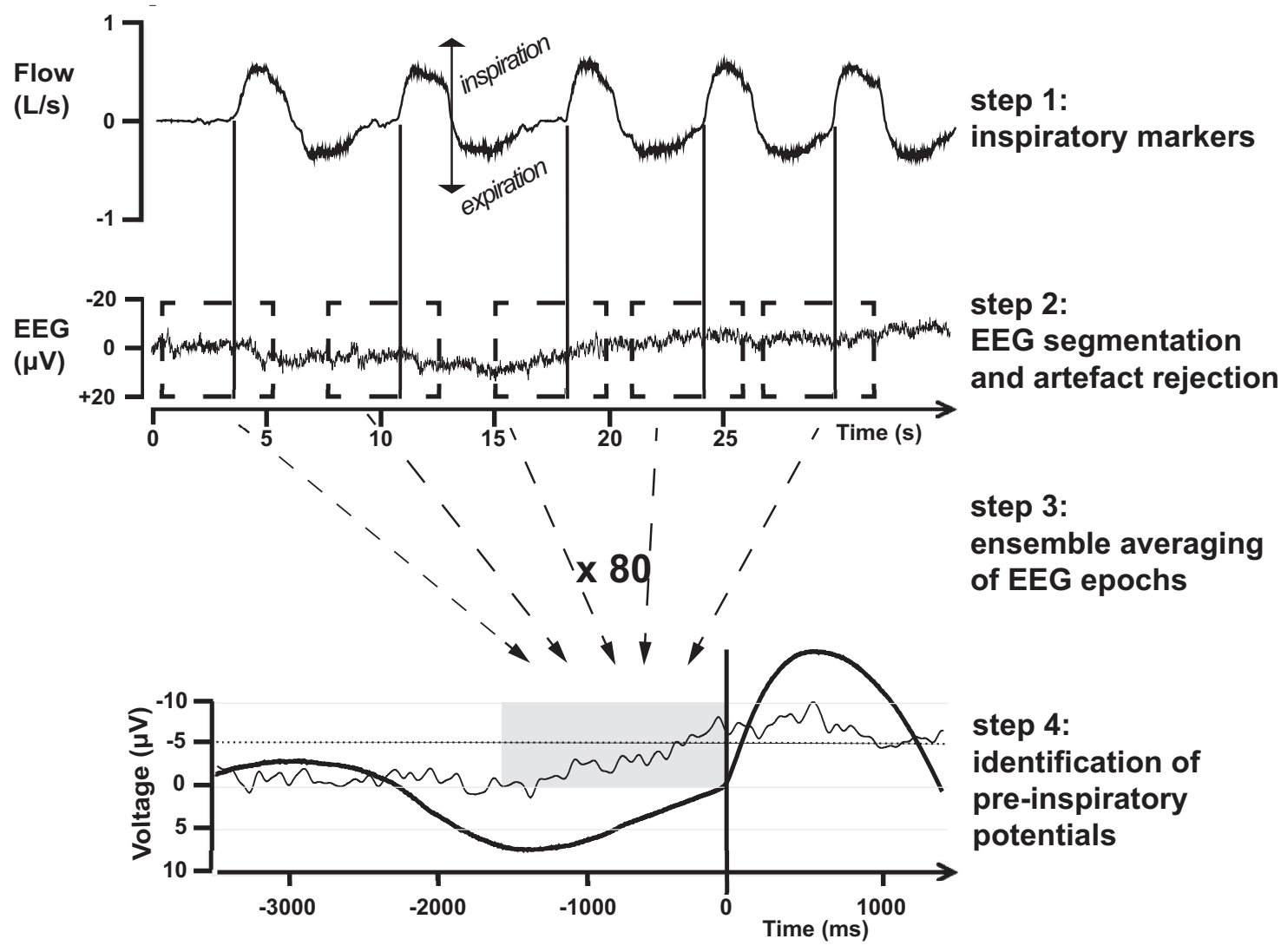

Figure 2. Identification of preinspiratory potentials. Simultaneous ventilation recordings (first plot at the top; inspiration toward the top, expiration toward the bottom) and electroencephalography (second plot) were used to identify preinspiratory potentials based on the following: (1) detection of the start of inspiration; (2) segmentation of the EEG based on this marker, followed by rejection of artifactual segments (see Materials and Methods); (3) averaging of $80 \mathrm{EEG}$ segments devoid of artifacts; and (4) visual identification of PIPs (see Materials and Methods) in the form of a negative wave (directed upwards) preceding inspiration.

the head, eyes, or limbs. No instructions were provided concerning breathing.

Recordings were performed under 4 successive experimental conditions (Fig. 1) as follows. Baseline spontaneous breathing recordings were initially performed under normocapnic conditions ( Pet $_{\mathrm{CO} 2}>35$ $\mathrm{mmHg}$ ). This session was designed to provide a negative control, as no breathing-related cortical activity is expected in this setting. Subjects then had to perform a series of rapid voluntary inspiratory maneuvers (sniffs). This session was designed to provide a positive control, as breathing-related cortical activity is expected in this setting (see below). Hypocapnia with $\mathrm{Pet}_{\mathrm{CO} 2}$ between 20 and $30 \mathrm{mmHg}$ was then induced passively by means of noninvasive ventilation delivered by a Servo 900C ventilator (Siemens). This ventilator was adjusted to deliver pressure-controlled cycles, with a fixed respiratory rate of $16 / \mathrm{min}$ and an inspiration/expiration ratio of 0.33 . Airway pressure was gradually increased to achieve a $\mathrm{Pet}_{\mathrm{CO} 2}$ of $20 \mathrm{mmHg}$ (median required value: $18 \mathrm{cmH}_{2} \mathrm{O}$ [interquartile range: 14-21] c). The ventilator was then disconnected from the circuit, and recordings were performed during hypocapnic spontaneous breathing (hypocapnia condition), defined as a $\mathrm{Pet}_{\mathrm{CO} 2}$ between 20 and $30 \mathrm{mmHg}$. If Pet $_{\mathrm{CO} 2}$ rose to $>30 \mathrm{mmHg}$ during the recording following passive hyperventilation, controlled ventilation was resumed to lower this value to $20 \mathrm{mmHg}$. This cycle was repeated as many times as necessary to allow the recording of 120 ventilatory cycles under hypocapnia (in practice, 3-8 times depending on the subject, with a median duration of ventilation of $17 \mathrm{~min} 50 \mathrm{~s}$ [ $13 \mathrm{~min} 59 \mathrm{~s}$ to $22 \mathrm{~min} 52 \mathrm{~s}$ ]). The median $\mathrm{Pet}_{\mathrm{CO} 2}$ during hypocapnia with this methodology was $26.9 \mathrm{mmHg}$ $(26.2-28 \mathrm{mmHg})$. The fourth and last condition consisted of recording during spontaneous breathing after $\mathrm{Pet}_{\mathrm{CO} 2}$ returned to a value $>35 \mathrm{mmHg}$ (washout). 
Data processing

Ventilation. The ventilatory flow signal was inspected to count the number of ventilatory cycles performed during spontaneous breathing in hypocapnia and any posthypocapnia apneas. Apnea was defined by prolongation of the expiratory time ( $\mathrm{TE}$ ) by $>3$ SDs compared with the Te measured under baseline conditions (Chenuel et al., 2006). The ventilatory behavior related to hypocapnia was characterized graphically and expressed by the ratio of the number of apneas over the number of ventilatory cycles. The number of apneas and ventilatory cycles taken into account in the calculation of this ratio corresponded to the median overall periods of induced hypocapnia. The minute ventilation $\left(\dot{\mathrm{V}}_{\mathrm{E}}\right)$, respiratory rate $\left(f_{\mathrm{R}}\right)$, and tidal volume $(\mathrm{VT})$ were calculated from the ventilatory flow signal (MATLAB 7, The MathWorks; RRID:SCR_001622).

Preinspiratory potentials. The reference EEG signal was defined as the linked ears reference (averaged between A1 and A2). The EEG signal was bandpass filtered between 0.05 and $10 \mathrm{~Hz}$. The start of inspiration was visually marked on the ventilatory flow signal to segment the EEG signal into synchronous segments of ventilation. Each segment began $3500 \mathrm{~ms}$ before the start of inspiration and ended $1500 \mathrm{~ms}$ after the start of inspiration. Under each condition, 120 segments were therefore generated and selected. Segments containing artifacts were rejected, where artifacts were defined as a variation of the signal by $>5 \mu \mathrm{V}$ per ms or $100 \mu \mathrm{V}$ over $200 \mathrm{~ms}$ or signals exceeding the limits of $100 \mu \mathrm{V}$. Artifacts were detected automatically on $\mathrm{Cz}, \mathrm{FCz}, \mathrm{Fz}, \mathrm{C} 3$, and $\mathrm{C} 4$ electrodes and then controlled visually. Eighty segments were then averaged point by point under each condition. The EEG reference line was then individually redefined (Analyzer 2.0, BrainProducts) (Fig. 2). Following this EEG processing, the primary endpoint was the presence or absence of a preinspiratory potential (PIP). The presence of a PIP was investigated visually by a first examiner, then confirmed or invalidated by a second examiner blinded to the test conditions. In the case of discordance between the two examiners, a third examiner was asked to decide. Visual identification of PIPs was based on morphological criteria, requiring the presence of a slow negative wave during the $2500 \mathrm{~ms}$ preceding inspiration, and topographical criteria (potential present in $\mathrm{Cz}$ and/or adjacent to $\mathrm{FCz}, \mathrm{Fz}, \mathrm{C} 3$, or $\mathrm{C} 4$ ). The latency of the PIP, defined as the time between the start of the negative deflection and the start of inspiration, and the amplitude of the PIP, defined as the maximum value of the EEG signal on the PIP before the start of inspiration, were then determined.

Statistical analysis (ventilatory data and preinspiratory potentials) All statistical analyses were performed with SPSS version 19 for Macintosh software (IBM; RRID:SCR_002865). The concordance between two examiners for identification of a PIP was evaluated by Cohen's $\kappa$ test. As the normality of distribution was not verified for all quantitative variables, these values were therefore expressed by their median and interquartile range and nonparametric statistical tests were used. The presence or absence of a PIP during hypocapnia between subjects with a PIP during hypocapnia and those without a PIP during hypocapnia was compared by Fisher's exact test. Comparisons between experimental conditions were performed exclusively in the group of subjects presenting a PIP during hypocapnia by Wilcoxon's and Friedman's tests. The equality of distribution of PIP for paired series was tested between all ventilation conditions and then by pairs using Cochran's Q test. Differences were considered to be significant for a $p$ value $<0.05$.
Time-frequency analysis

In addition to the PPI analysis described above, respiratory-related cortical activity was studied using time-frequency analysis for corroboratory purposes, as follows.

Time-frequency maps. For each $s(t)$ signal, EEG recording were analyzed in the time-frequency domain by convolution with complex Gaussian Morlet's wavelets $w(t, f)$ (Tallon-Baudry et al., 1997), thus providing a time-frequency power map $P(t, f)=|w(t, f) \times s(t)|^{2}$, where $w(t, f)$ is a complex Morlet's wavelet as follows:

$$
w(t, f)=A \cdot \exp \left(-t^{2} / 2 \sigma_{t}^{2}\right) \cdot \exp (2 i \pi f t)
$$

with $A=\left(\sigma_{t} \sqrt{\pi}\right)^{-1 / 2}$ and $\sigma_{f}=1 /\left(2 \pi \sigma_{t}\right)$ a function of the frequency $f$ : $\sigma_{f}=f / 7$.

Normalized time-frequency maps were computed for each EEG recording for visualization purposes. This normalization was performed separately for each frequency and consisted of (1) subtracting the mean power during a prestimulus baseline ( $-1900 \mathrm{~ms}:-1600 \mathrm{~ms})$ and (2) dividing by the SD of the power during this same baseline. Timefrequency analysis was performed by computation of $P(t, f)$ on the preinspiratory potential for time-locked activities linked to the respiration signal.

\section{Statistical analysis of time-frequency maps}

Significance of the differences of all contrasts was established using a nonparametric cluster randomization test across spatial and spectral domains (Maris and Oostenveld, 2007). This test effectively controls the false discovery rate in situations involving multiple comparisons by clustering neighboring quantities that exhibit the same effect. For the power map $P(t, f)$ analysis, the neighborhood was univariate across time (adjacent time-sample over the whole time-window) and frequencies (sideby-side frequency bins). The permutation method exhibits values for which $t$ statistics exceeded a given critical value when comparing two conditions value by value. To correct for multiple comparisons, neighbor 


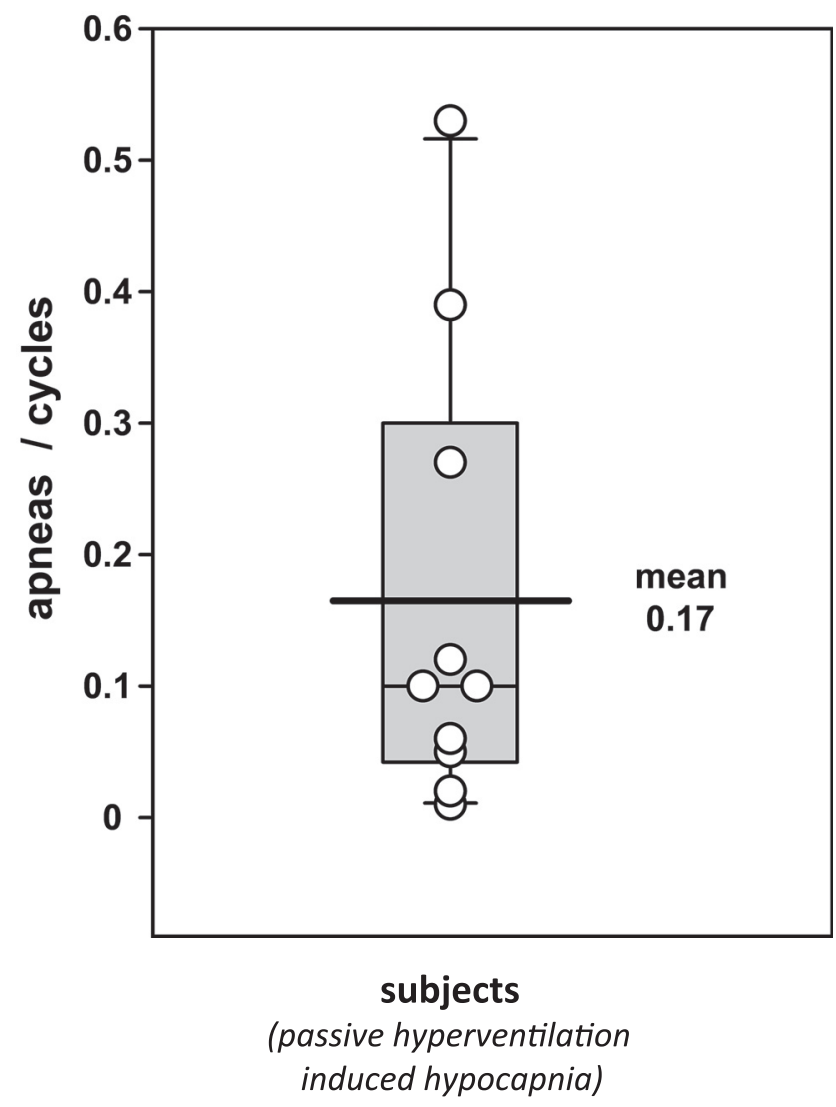

Figure 4. Proportion of apneic cycles during the hypocapnic breathing phase of the protocol. The top 3 subjects exhibited a hypocapnia-related apneic pattern and did not present preinspiratory potentials during the hypocapnia period. The bottom 7 subjects did not exhibit hypocapnia-related apneas; 6 of them presented preinspiratory potentials during the hypocapnia period.

values exceeding the critical value were considered to be members of the same cluster. The cluster-statistic (CS) was taken as the sum of $t$ values in a given cluster. The false discovery rate was controlled by evaluating the CS distribution on 1000 permutations (Pantazis et al., 2005). Each permutation represented a randomization of the data between the two conditions and across multiple subjects. For each permutation, the CSs were computed by taking the cluster with the maximum sum of $t$ statistics. The threshold that controls the family-wise error rate was determined according to the proportion of the randomization null distribution exceeding the observed maximum CS (Monte Carlo test). We used a threshold critical value of $3 \sigma$. Statistics were then corrected for both time and spectral dimensions, and the significance of difference of contrasts was determined for rejection of the null hypothesis and control of the false alarm rate at $p=0.01$.

\section{Complementary set of experiments (hypocapnia induced by active hyperventilation)}

A second series of experiments was conducted on a separate set of 10 subjects ( 4 men, 6 women; median age 37 years, range $24-51$ years) during a separate experimental campaign, for corroboratory purposes. The experimental setup was simplified, but globally similar to that used during the main set of experiments, with ventilatory measurements being performed either with small and unobtrusive nasal probes or with a pair of magnetometers (Mead et al., 1967) placed at the level of the umbilicus. The experimental protocol consisted of normocapnic ventilation and hypocapnic ventilation only; hypocapnia was induced by voluntary hyperventilation. Because of the methodological differences between the two sets of experiments, the EEG recordings were not pooled for ensemble averaging.
Table 1. Breathing pattern in the 7 subjects not exhibiting hypocapnia-induced apneas $^{a}$

\begin{tabular}{lcccl}
\hline & Baseline & Hypocapnia & Washout & $p$ \\
\hline $\mathrm{V}_{\mathrm{T}}(\mathrm{ml})$ & $397(344-420)$ & $386(180-455)$ & $328(278-363)$ & $0.0162^{*}$ \\
$f_{\mathrm{R}}(/ \mathrm{min})$ & $17(15-18)$ & $17(16-21)$ & $17(13-20)$ & $\mathrm{NS}$ \\
$\mathrm{V}_{\mathrm{E}}(\mathrm{L} / \mathrm{min})$ & $6.14(4.73-7.02)$ & $5.33(2.57-7.27)$ & $4.73(3.33-5.44)$ & $\mathrm{NS}$ \\
\hline
\end{tabular}

${ }^{a}$ Data are median (interquartile range). $V_{T}$, Tidal volume; $f_{R}$, respiratory rate; $V_{E}$, minute ventilation.

*Significant difference between baseline and washout only, not between baseline and hypocapnia.

\section{Results}

Main set of experiments (hypocapnia induced by passive hyperventilation)

Breathing pattern in hypocapnia

Visual inspection of the ventilatory flow recordings indicated the presence of an apneic breathing pattern in 3 subjects and the absence of this pattern in 7 subjects (Fig. 3). According to the above definition (see Materials and Methods), the median apneic cycles/total cycles ratio in the 10 participating subjects was 0.10 (0.04-0.33), with a mean value of 0.17 that clearly distinguished the two groups of individuals (Fig. 4). The breathing pattern characteristics of the 7 subjects who did not exhibit hypocapniarelated apneas are described in Table 1. No significant differences were observed between the baseline breathing pattern and the hypocapnic breathing pattern in terms of tidal volume, breathing frequency, or ventilation.

\section{Preinspiratory potentials}

As expected, all subjects exhibited clear and typical preinspiratory potentials during the sniff condition. The interobserver $\kappa$ coefficient for all aggregated conditions was 0.85 .

In the 3 subjects who exhibited a hypocapnia-induced apneic breathing pattern, no preinspiratory potential was visible during baseline, hypocapnia, or washout. In the 7 subjects who did not exhibit a hypocapnia-induced apneic breathing pattern, a preinspiratory potential was visible during baseline in one case and during washout in one other case. A preinspiratory potential was present in 6 cases during hypocapnia ( $p=0.033$ in comparison with the subjects exhibiting an apneic breathing pattern). The subject in whom no preinspiratory potential was present during hypocapnia did not exhibit any preinspiratory potential during baseline or washout (Figs. 5, 6).

In the 7 subjects who did not exhibit a hypocapnia-induced apneic breathing pattern, preinspiratory potentials were significantly more frequent during hypocapnia than during baseline or washout ( $p=0.037)$. Excluding the two subjects with preinspiratory potentials during baseline and washout did not modify this result.

The latencies and amplitudes of the preinspiratory potentials observed during hypocapnia were $1031 \mathrm{~ms}$ (767-1666) and 6.22 $\mu \mathrm{V}$ (4.76-7.17), not significantly different from those observed during sniffs in the same subjects (latency $1572 \mathrm{~ms}$ [1070-1781], $p=0.184$; amplitude $9.28 \mu \mathrm{V}[8.13-15.2], p=0.120)$.

\section{Time-frequency maps}

In all subjects, voluntary inspirations induced an increase in $\delta$ amplitudes in the frontal, frontocentral, and central regions $(\mathrm{Fz}$, $\mathrm{FCz}, \mathrm{Cz}, 0.6-1 \mathrm{~Hz}$; $-1500 \mathrm{~ms}$ to $-510 \mathrm{~ms}$, average CS = $-1030, p<0.01)$ and also an increase in $\beta$ rhythm in the central region ( $\mathrm{Fz}, 20-23 \mathrm{~Hz} ;-385 \mathrm{~ms}$ to $-350 \mathrm{~ms}$, CS $=-443, p<$ 0.01 ) in comparison with quiet breathing (sniffs vs baseline).

In the 6 subjects who did not exhibit a hypocapnia-induced apneic breathing pattern and who exhibited a preinspiratory potential during hypocapnia, contrasting the time-frequency 
maps showed a marked increase in the $\delta$ band amplitude on the $\mathrm{FCz}$ electrode during hypocapnia compared with baseline $(0.6-1 \mathrm{~Hz}$; -1350 to $420 \mathrm{~ms}$, CS $=-1071, p<0.01)$ (Fig. 7).

\section{Complementary set of experiments (hypocapnia induced by active hyperventilation)}

Nine of the 10 datasets from the subjects participating in this set of experiments were analyzed (one EEG recording was discarded because of continuous noise). None of these subjects exhibited preinspiratory potentials during normocapnic breathing. During hypocapnic breathing, 6 subjects maintained nonapneic ventilation and exhibited preinspiratory potentials, whereas the other 3 subjects exhibited apneas with no preinspiratory potentials.

Pooling the two sets of experiments confirmed a highly significant difference in the frequency of preinspiratory potentials between the 13 subjects without hypocapnia-induced apneas and the 6 subjects with hypocapnia-induced apneas $\left(p<10^{-3}\right)$. Pooled analysis also confirmed that, in the 13 subjects without hypocapnia-induced apneas, preinspiratory potentials were significantly more frequent during hypocapnia than during normocapnia $\left(p<10^{-3}\right)$.

\section{Discussion}

By showing that subjects who maintain a ventilatory activity despite hypocapnia exhibit a respiratory-related cortical activity, while such activity is absent in subjects who exhibit hypocapniarelated apneas, this study provides a neurophysiological substrate for the peculiarity that constitutes the resistance of ventilation to hypocapnia in awake humans.

\section{Methodological considerations}

Studies of hypocapnic breathing have placed considerable emphasis on the hypocapnia-inducing procedure. After active hyperventilation, hypocapnic breathing can be explained by posthyperventilation facilitation mechanisms involving the medulla, as they are present in decorticated animals (Eldridge, 1976). These mechanisms cannot explain our observations when hypocapnia was induced by passive hyperventilation, except if our subjects failed to actually relax under mechanical ventilation. In this case, it could be argued that the respiratory-related cortical activity observed after passive hyperventilation was a carryover of an activity elicited by the hypocapniainducing procedure itself. During preliminary experiments, we did observe preinspiratory potentials during mechanical ventilation (data not shown) that could have been related to either hypocapnia or incomplete relaxation. We therefore conducted control experiments during which passive hyperventilation was associated with $\mathrm{CO}_{2}$ administration to maintain isocapnia (data not shown): no preinspiratory potentials were observed. It has been postulated that the stimulus provided by the mouthpiece and nose clip used to measure ventilation could account for the persistence of ventilation after passive hypoventilation (Corfield et al., 1995). In our complementary set of experiments, ventilatory activity was assessed without a mouthpiece (see Materials and Methods), and the observations were identical. We found a strict parallelism between posthyperventilation preinspiratory potentials and apneas after passive and voluntary

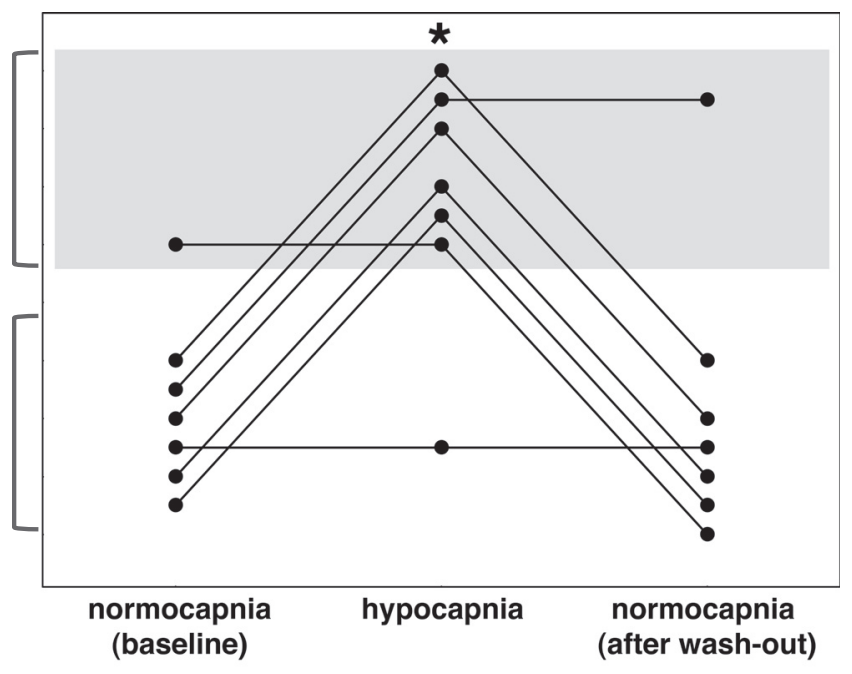

hyperventilation. Hypocapnic preinspiratory potentials consistently disappeared after return of $\mathrm{Pa}_{\mathrm{CO} 2}$ to baseline, a strong argument against the potential biases described above that is also suggestive of causality. Of note, the first description of respiratory premotor potentials (Macefield and Gandevia, 1991) mentioned the absence of cortical activity in one subject breathing in hypocapnia after voluntary hyperventilation. This subject was recorded $3 \mathrm{~min}$ at a $\mathrm{Pa}_{\mathrm{CO} 2}$ of $28-30 \mathrm{mmHg}$, versus $10 \mathrm{~min}$ at lower $\mathrm{Pa}_{\mathrm{CO} 2}$ in our case, which might account for the difference. Electroencephalographic recordings are less precise measures of cortical activity than intracranial recordings or functional neuroimaging studies. However, cortical signals detected by scalp EEG by nature reflect intense activities; and by using event-related approaches with thorough preprocessing to eliminate artifacts, we optimized the signal-to-noise ratio and ensured that the activity detected was respiratory specific. In addition, we presented results averaged over the study population, which is a very conservative approach. Both the signal processing approaches (premotor potentials and time-frequency maps) that we used have been extensively validated before. The time-frequency analysis is particularly apt at identifying an event with a given frequency among artifacts of different frequencies, and does so with a high degree of precision allowed by the wavelet approach. In our subjects, the timefrequency analysis (Fig. 7) fully corroborated the preinspiratory potentials analysis by confirming that cortical activity changed during the preinspiratory period during hypocapnic breathing compared with normocapnic breathing, mostly in the $\delta$ frequency band. These findings are similar to those obtained during experimentally loaded breathing in normal subjects (Hudson et al., 2016). All in all, we believe that our results are neurophysiologically robust and do demonstrate a cortical contribution to the neural respiratory drive to breathe in hypocapnia.

\section{Physiological significance}

The contrast between sleep and wakefulness regarding hypocapnic breathing strongly contributed to the concept of human wakefulness drive to breathe. Identifying a cortical substrate to the hypocapnic breathing drive is therefore not unexpected. The cortical activity that we observed is compatible with SMA involvement, resembling that observed in reaction to inspiratory loading (Raux et al., 2007, 2013; Hudson et al., 2016). Of note, the paradoxical hyperventilation in response to inspiratory loading 

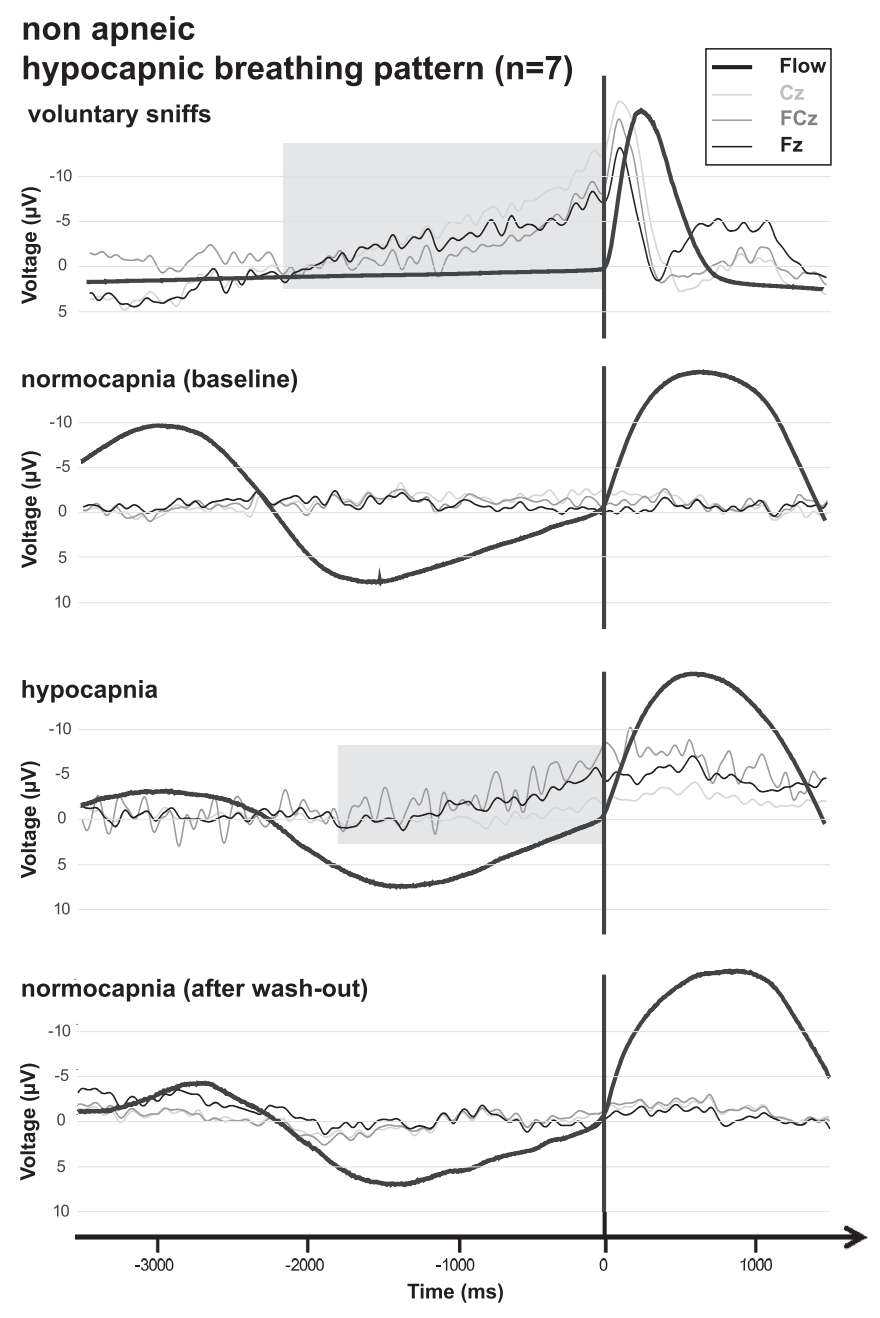

Figure 6. Ensemble averaging of the signals obtained in the 4 different experimental conditions, in the 7 subjects not exhibiting hypocapnia-induced apneas (left) and the 3 subjects exhibiting hypocapnia-induced apneas (right). Vertical bar represents the beginning of inspiration as identified on the flow recording. Gray areas represent preinspiratory potentials when present. In each condition, the Cz (light gray), FCz (medium gray), and Fz (dark gray) electrodes are represented on the EEG recording.

(Yanos et al., 1990) also depends on wakefulness (Pillar et al., 2000). In both situations (hypocapnic breathing and inspiratory loading), the loss of an SMA influence on breathing during sleep could be due to a sleep-related breakdown in cortical connectivity (Massimini et al., 2005). Sleep depresses the excitability of the corticospinal pathway to the diaphragm (Mehiri et al., 2006), as does inhibitory conditioning of the SMA by repetitive transcranial magnetic stimulation (Laviolette et al., 2013).

Several mechanisms can be proposed to explain the respiratoryrelated cortical activation in hypocapnia. One would be the activation of a cortical oscillator when medullary oscillators are extinguished by hypocapnia. Haouzi and Bell (2009) and Haouzi et al. (2007) provided arguments in favor of this possibility by suggesting the existence of cortically set programs able to generate regulatory combinations of respiratory cycle duration and magnitudes to maintain Pet $_{\mathrm{CO} 2}$ constant or almost constant during cortically driven breathing. Raux et al. (2013) suggested that cortically driven breathing can be automatized during inspiratory loading. Alternatively, activation of cortical structures could maintain hypocapnic breathing via corticosubcortical cooperation. Descending respiratory outputs (from medullary structures, limbic structures, or cortical structures) and afferent respiratory and nonrespiratory inputs converge onto respiratory spinal motoneurons and all contribute to determine their excitability, namely, their responsiveness to any type

\section{apneic}
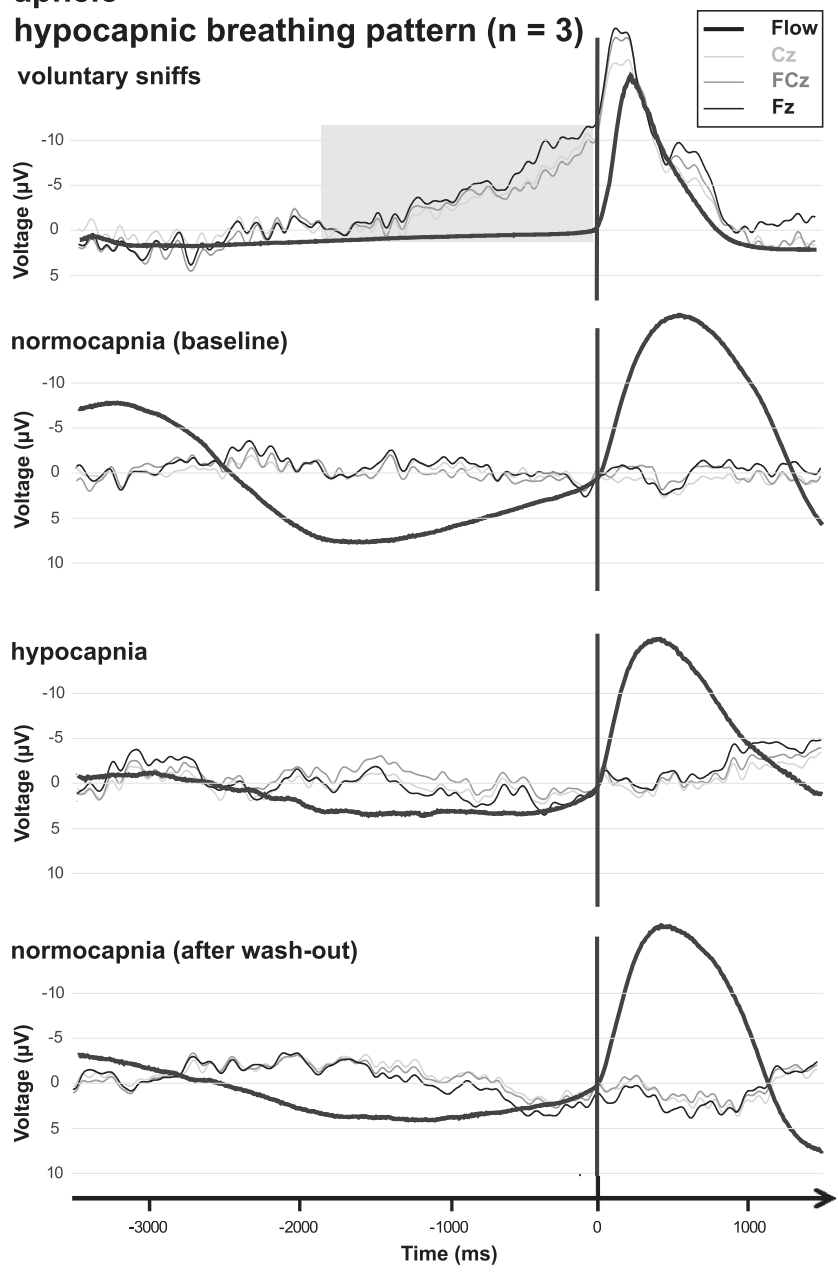

of stimulus. As a result, the various components of the neural respiratory drive interact with one another and generally facilitate each other (e.g., enhanced diaphragm response to a corticospinal volley in conjunction with a $\mathrm{CO}_{2}$ enhanced bulbospinal outflow) (Straus et al., 2004). In the case of hypocapnic breathing, the cortical activation that we describe could sensitize respiratory spinal motoneurons to the hypocapnia-depressed medullary drive to breathe. This is the same hypothesis that has been elaborated to explain the wakefulnessrelated maintenance of ventilation in patients with congenital central hypoventilation syndrome who do exhibit a respiratory-related cortical activity (Tremoureux et al., 2014a). Interindividual differences regarding hypocapnia-induced apneas could derive from a variable impact of hypocapnia on medullary respiratory generators. Of note, we observed preinspiratory potentials after both passive and active hyperventilation, which suggests that hypocapnic breathing after active hyperventilation could proceed from several, nonmutually exclusive mechanisms, including medullary after-discharge (Eldridge, 1973, 1974, 1976; Corfield et al., 1995) and cortical activation.

It has yet to be determined how the cortical network contributing to hypocapnic breathing becomes activated. Hypocapnia per se could be involved. The afferent stimulations associated with the hypocapnia-inducing procedure could also be involved. Jack et al. (2010) submitted patients with chronic hyperventilation to transient 
inspiratory occlusions and observed that the resulting cerebral activation was more marked in these patients than in control subjects. Not unexpectedly, this augmented activation was observed in the anterior insula that is known to be involved in the monitoring of respiratory-related afferent signals to the brain. Less expectedly, augmented activation was also present in premotor and motor areas, which is compatible with triggering of an (exaggerated) motor response by the afferent stimulus. Finally, the sudden cessation in respiratory movements at the end of the hypocapniainducing procedure could also be the trigger for the corresponding respiratory-related cortical activity, but the time resolution of the EEG approaches used in this study is insufficient to test this hypothesis. Examining hypocapnic breathing recordings available in the literature suggests that, after passive hyperventilation, a single brief apnea can precede the hypocapnic ventilatory activity (Bainton and Mitchell, 1966; Corfield et al., 1995). One indirect argument in favor of the role of respiratory afferents in activation of the cortical network responsible for hypocapnic breathing (as opposed to hypocapnia itself) derives from the observations by Bainton and Mitchell (1966) that repeating passive hyperventilation over several consecutive days results in attenuation of the hypocapnic breathing phenomenon (more frequent apneic responses), namely, an habituation dynamic similar to that observed during inspiratory loading (Gozal et al., 1995).

In conclusion, the respiratory-related EEG activity observed in this study resembles the respiratory-related activity that has been described during experimental inspiratory loading (Raux et al., 2007), during experimental expiratory loading (Morawiec et al., 2015), during speech (Tremoureux et al., 2014b), in sleep apnea patients with intrinsically increased upper airway resistance (Launois et al., 2015), in amyotrophic lateral sclerosis patients with weak respiratory muscles (Georges et al., 2016), and in patients with defective automatic breathing control (Tremoureux et al., 2014a). Together, these results suggest the existence of a general mechanism of corticosubcortical cooperation to generate the drive to breathe in awake humans, involving neural structures that source premotor potentials, including the SMA (Lee et al., 1986; Tarkka and Hallett, 1990; Ball et al., 1999). This hypothesis is in line with the known respiratory connections of the SMA, both efferent (Sharshar et al., 2004; Raux et al., 2010, 2013; Laviolette et al., 2013) and afferent (Logie et al., 1998; von Leupoldt et al., 2010). From an evolutionary perspective, the ability to supervene the automatic control of breathing by voluntary or automatized cortical mechanisms is a prerequisite for the full development of speech. Without this ability, ut-

\section{A normocapnic breathing (baseline) - FCz derivation}

\section{1) average time-frequency map before baseline correction}

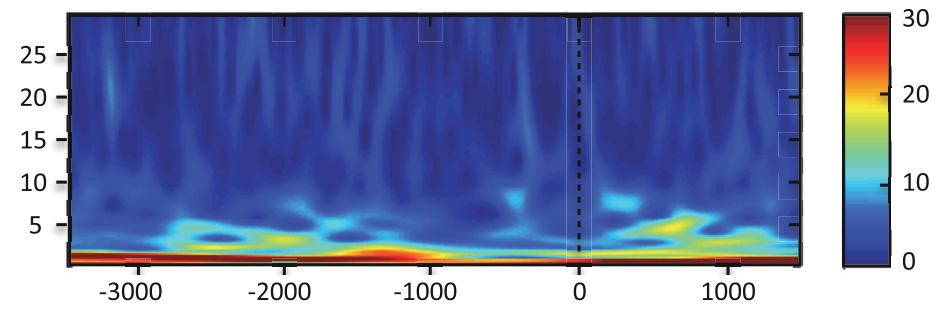

$\frac{\sqrt{3}}{3}$

2) average time-trequency map after baseline correction

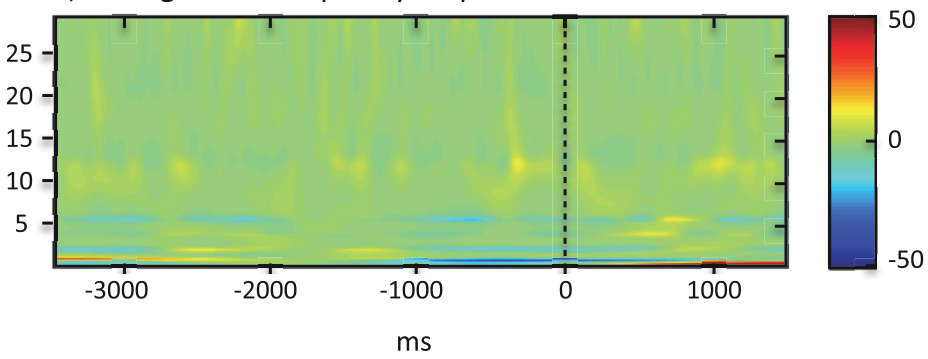

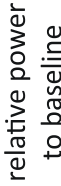

\section{B hypocapnic breathing - FCz derivation}

1) average time-frequency map before baseline correction

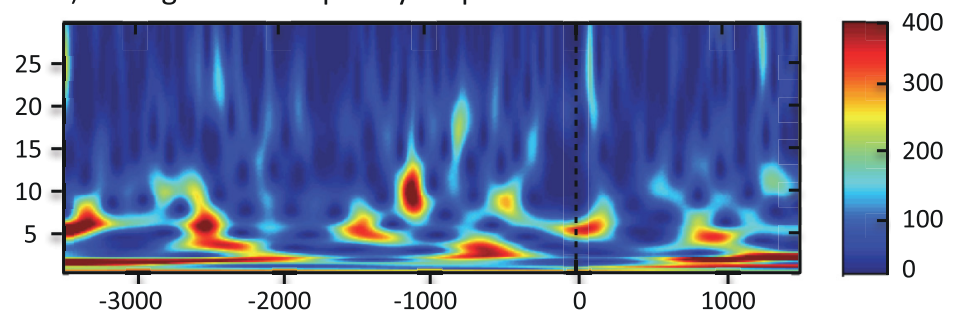

2) average time-frequency map after baseline correction

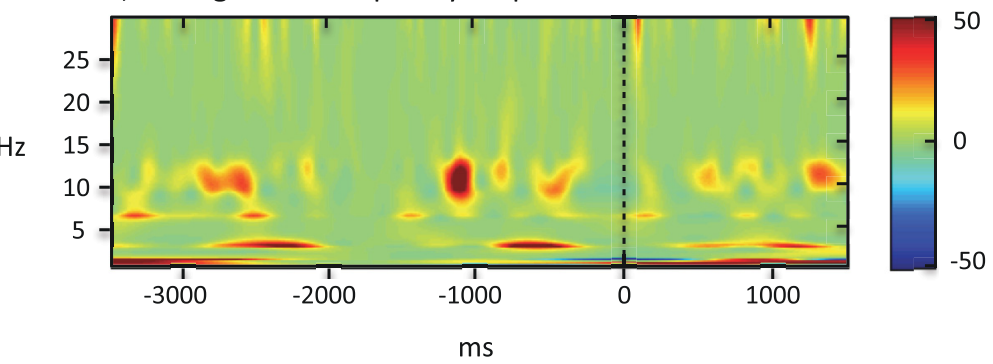

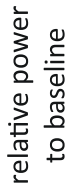

Figure 7. Time-frequency maps of respiratory-related cortical activity in the FCz derivation. Ensemble averaging of the eventrelated time-frequency T-F maps obtained during $(\boldsymbol{A})$ normocapnic breathing and $(\boldsymbol{B})$ hypocapnic breathing. T-F maps highlight synchronized oscillations (across trials) with respect to inspiratory onset (vertical lines at $t=0 \mathrm{~s}$ ). $\boldsymbol{A} \mathbf{1}, \boldsymbol{B} \mathbf{1}$, Total average EEG power at different time-frequency pairs. $\mathbf{A 2}, \mathbf{B 2}$, Relative increase of power after correcting the baseline (see Materials and Methods). Red colored zones represent an increased cortical activity during hypocapnic breathing at a given time and frequency.

terance modulation and prosody would be very limited. This is well illustrated by the restricted speech capacities of mechanically ventilated patients who cannot control automatic breathing, as breathing rhythm is determined by an external machine. These patients can speak but have to adapt the length of their sentences to the set breathing frequency, and they cannot vary the loudness of their utterances (Hoit et al., 1994). Of note, regardless of the evolutionary driver of the cortical control of breathing, its establishment must predate the development of speech itself. From a clinical perspective, the present results are an additional incentive to study the cortical control of breathing during certain speech 
disorders (Tremoureux et al., 2014b). A dysregulation of the hypocapnia-induced respiratory-related cortical activity described here could be involved in patient suffering from idiopathic chronic hyperventilation (who do not hyperventilate during sleep) (Gardner et al., 1986). Identifying a respiratoryrelated cortical activity during spontaneous breathing could be of diagnostic value in this setting.

\section{References}

Bainton CR, Mitchell RA (1966) Posthyperventilation apnea in awake man. J Appl Physiol 21:411-415. Medline

Ball T, Schreiber A, Feige B, Wagner M, Lücking CH, Kristeva-Feige R (1999) The role of higher-order motor areas in voluntary movement as revealed by high-resolution EEG and fMRI. Neuroimage 10:682-694. CrossRef Medline

Bunn JC, Mead J (1971) Control of ventilation during speech. J Appl Physiol 31:870-872. Medline

Chenuel BJ, Smith CA, Skatrud JB, Henderson KS, Dempsey JA (2006) Increased propensity for apnea in response to acute elevations in left atrial pressure during sleep in the dog. J Appl Physiol 101:76-83. CrossRef Medline

Corfield DR, Morrell MJ, Guz A (1995) The nature of breathing during hypocapnia in awake man. Respir Physiol 101:145-159. CrossRef Medline

Datta AK, Shea SA, Horner RL, Guz A (1991) The influence of induced hypocapnia and sleep on the endogenous respiratory rhythm in humans. J Physiol 440:17-33. CrossRef Medline

Eldridge FL (1973) Posthyperventilation breathing: different effects of active and passive hyperventilation. J Appl Physiol 34:422-430. Medline

Eldridge FL (1974) Central neural respiratory stimulatory effect of active respiration. J Appl Physiol 37:723-735. Medline

Eldridge FL (1976) Central neural stimulation of respiration in unanesthetized decerebrate cats. J Appl Physiol 40:23-28. Medline

Fauroux B, Isabey D, Desmarais G, Brochard L, Harf A, Lofaso F (1998) Nonchemical influence of inspiratory pressure support on inspiratory activity in humans. J Appl Physiol 85:2169-2175. Medline

Feldman JL, Del Negro CA, Gray PA (2013) Understanding the rhythm of breathing: so near, yet so far. Annu Rev Physiol 75:423-452. CrossRef Medline

Fink BR (1961) Influence of cerebral activity in wakefulness on regulation of breathing. J Appl Physiol 16:15-20. Medline

Gandevia SC, Rothwell JC (1987) Activation of the human diaphragm from the motor cortex. J Physiol 384:109-118. CrossRef Medline

Gardner WN, Meah MS, Bass C (1986) Controlled study of respiratory responses during prolonged measurement in patients with chronic hyperventilation. Lancet 2:826-830. CrossRef Medline

Georges M, Moraviec E, Raux M, Gonzalez-Bermejo J, Pradat PF, Similowski T, Morélot-Panzini C (2016) Cortical drive to breathe in amyotrophic lateral sclerosis: a dyspnoea-worsening defence? Eur Respir J 47:18181828. CrossRef Medline

Gozal D, Omidvar O, Kirlew KA, Hathout GM, Hamilton R, Lufkin RB, Harper RM (1995) Identification of human brain regions underlying responses to resistive inspiratory loading with functional magnetic resonance imaging. Proc Natl Acad Sci U S A 92:6607-6611. CrossRef Medline

Hanks EC, Ngai SH, Fink BR (1961) The respiratory threshold for carbon dioxide in anesthetized man: determination of carbon dioxide threshold during halothane anesthesia. Anesthesiology 22:393-397. CrossRef Medline

Haouzi P, Bell HJ (2009) Control of breathing and volitional respiratory rhythm in humans. J Appl Physiol 106:904-910. CrossRef Medline

Haouzi P, Chenuel B, Whipp BJ (2007) Control of breathing during cortical substitution of the spontaneous automatic respiratory rhythm. Respir Physiol Neurobiol 159:211-218. CrossRef Medline

Henke KG, Arias A, Skatrud JB, Dempsey JA (1988) Inhibition of inspiratory muscle activity during sleep: chemical and nonchemical influences. Am Rev Respir Dis 138:8-15. CrossRef Medline

Hoit JD, Shea SA, Banzett RB (1994) Speech production during mechanical ventilation in tracheostomized individuals. J Speech Hear Res 37:53-63. CrossRef Medline

Hopkinson NS, Sharshar T, Dayer MJ, Lofaso F, Moxham J, Polkey MI (2012) The effect of acute non-invasive ventilation on corticospinal pathways to the respiratory muscles in chronic obstructive pulmonary disease. Respir Physiol Neurobiol 183:41-47. CrossRef Medline

Hudson AL, Navarro-Sune X, Martinerie J, Pouget P, Raux M, Chavez M, Similowski T (2016) Electroencephalographic detection of respiratoryrelated cortical activity in humans: from event-related approaches to continuous connectivity evaluation. J Neurophysiol 115:2214-2223. CrossRef Medline

Jack S, Kemp GJ, Bimson WE, Calverley PM, Corfield DR (2010) Patterns of brain activity in response to respiratory stimulation in patients with idiopathic hyperventilation (IHV). Adv Exp Med Biol 669:341-345. CrossRef Medline

Launois C, Attali V, Georges M, Raux M, Morawiec E, Rivals I, Arnulf I, Similowski T (2015) Cortical drive to breathe during wakefulness in patients with obstructive sleep apnea syndrome. Sleep 38:1743-1749. CrossRef Medline

Laviolette L, Niérat MC, Hudson AL, Raux M, Allard E, Similowski T (2013) The supplementary motor area exerts a tonic excitatory influence on corticospinal projections to phrenic motoneurons in awake humans. PLoS One 8:e62258. CrossRef Medline

Lee BI, Lüders H, Lesser RP, Dinner DS, Morris HH 3rd (1986) Cortical potentials related to voluntary and passive finger movements recorded from subdural electrodes in humans. Ann Neurol 20:32-37. CrossRef Medline

Logie ST, Colrain IM, Webster KE (1998) Source dipole analysis of the early components of the RREP. Brain Topogr 11:153-164. CrossRef Medline

Macefield G, Gandevia SC (1991) The cortical drive to human respiratory muscles in the awake state assessed by premotor cerebral potentials. J Physiol 439:545-558. CrossRef Medline

Maris E, Oostenveld R (2007) Nonparametric statistical testing of EEG- and MEG-data. J Neurosci Methods 164:177-190. CrossRef Medline

Massimini M, Ferrarelli F, Huber R, Esser SK, Singh H, Tononi G (2005) Breakdown of cortical effective connectivity during sleep. Science 309: 2228-2232. CrossRef Medline

Mead J, Peterson N, Grimby G (1967) Pulmonary ventilation measured from body surface movements. Science 156:1383-1384. CrossRef Medline

Mehiri S, Straus C, Arnulf I, Attali V, Zelter M, Derenne JP, Similowski T (2006) Responses of the diaphragm to transcranial magnetic stimulation during wake and sleep in humans. Respir Physiol Neurobiol 154:406418. CrossRef Medline

Morawiec E, Raux M, Kindler F, Laviolette L, Similowski T (2015) Expiratory load compensation is associated with electroencephalographic premotor potentials in humans. J Appl Physiol 118:1023-1030. CrossRef Medline

Nuding SC, Segers LS, Iceman KE, O'Connor R, Dean JB, Bolser DC, Baekey DM, Dick TE, Shannon R, Morris KF, Lindsey BG (2015) Functional connectivity in raphe-pontomedullary circuits supports active suppression of breathing during hypocapnic apnea. J Neurophysiol 114:21622186. CrossRef Medline

Pantazis D, Nichols TE, Baillet S, Leahy RM (2005) A comparison of random field theory and permutation methods for the statistical analysis of MEG data. Neuroimage 25:383-394. CrossRef Medline

Pillar G, Malhotra A, Fogel R, Beauregard J, Schnall R, White DP (2000) Airway mechanics and ventilation in response to resistive loading during sleep: influence of gender. Am J Respir Crit Care Med 162:1627-1632. CrossRef Medline

Raux M, Straus C, Redolfi S, Morelot-Panzini C, Couturier A, Hug F, Similowski T (2007) Electroencephalographic evidence for pre-motor cortex activation during inspiratory loading in humans. J Physiol 578: 569-578. CrossRef Medline

Raux M, Xie H, Similowski T, Koski L (2010) Facilitatory conditioning of the supplementary motor area in humans enhances the corticophrenic responsiveness to transcranial magnetic stimulation. J Appl Physiol 108: 39-46. CrossRef Medline

Raux M, Tyvaert L, Ferreira M, Kindler F, Bardinet E, Karachi C, MorelotPanzini C, Gotman J, Pike GB, Koski L, Similowski T (2013) Functional magnetic resonance imaging suggests automatization of the cortical response to inspiratory threshold loading in humans. Respir Physiol Neurobiol 189:571-580. CrossRef Medline

Rektor I (2002) Scalp-recorded Bereitschaftspotential is the result of the activity of cortical and subcortical generators: a hypothesis. Clin Neurophysiol 113:1998-2005. CrossRef Medline 
Sharshar T, Hopkinson NS, Jonville S, Prigent H, Carlier R, Dayer MJ, Swallow EB, Lofaso F, Moxham J, Polkey MI (2004) Demonstration of a second rapidly conducting cortico-diaphragmatic pathway in humans. J Physiol 560:897-908. CrossRef Medline

Shea SA (1996) Behavioural and arousal-related influences on breathing in humans. Exp Physiol 81:1-26. CrossRef Medline

Similowski T, Straus C, Coïc L, Derenne JP (1996) Facilitation-independent response of the diaphragm to cortical magnetic stimulation. Am J Respir Crit Care Med 154:1771-1777. CrossRef Medline

Smith WR (1938) The representation of respiratory movements in the cerebral cortex. J Neurophysiol 1:55-68.

Straus C, Locher C, Zelter M, Derenne JP, Similowski T (2004) Facilitation of the diaphragm response to transcranial magnetic stimulation by increases in human respiratory drive. J Appl Physiol 97:902-912. CrossRef Medline

Sun Q, Goodchild AK, Pilowsky PM (2001) Firing patterns of pre-Botzinger and Botzinger neurons during hypocapnia in the adult rat. Brain Res 903:198-206. CrossRef Medline

Tallon-Baudry C, Bertrand O, Delpuech C, Permier J (1997) Oscillatory gamma-band $(30-70 \mathrm{~Hz})$ activity induced by a visual search task in humans. J Neurosci 17:722-734. Medline

Tarkka IM, Hallett M (1990) Cortical topography of premotor and motor potentials preceding self-paced, voluntary movement of dominant and non-dominant hands. Electroencephalogr Clin Neurophysiol 75:36-43. CrossRef Medline

Tremoureux L, Raux M, Hudson AL, Ranohavimparany A, Straus C, Similowski T (2014a) Does the supplementary motor area keep patients with Ondine's curse syndrome breathing while awake? PLoS One 9:e84534. CrossRef Medline

Tremoureux L, Raux M, Ranohavimparany A, Morélot-Panzini C, Pouget P, Similowski T (2014b) Electroencephalographic evidence for a respiratory-related cortical activity specific of the preparation of prephonatory breaths. Respir Physiol Neurobiol 204:64-70. CrossRef Medline

van Dixhoorn J, Duivenvoorden HJ (1985) Efficacy of Nijmegen Questionnaire in recognition of the hyperventilation syndrome. J Psychosom Res 29:199-206. CrossRef Medline

van Dixhoorn J, Folgering H (2015) The Nijmegen questionnaire and dysfunctional breathing. ERJ Open Reserach 1:00001-02015.

von Leupoldt A, Keil A, Chan PY, Bradley MM, Lang PJ, Davenport PW (2010) Cortical sources of the respiratory-related evoked potential. Respir Physiol Neurobiol 170:198-201. CrossRef Medline

Yanos J, Banner A, Stanko R, Gentry S, Greenawalt K (1990) Ventilatory responses to inspiratory threshold loading in humans. J Appl Physiol 68:2511-2520. Medline 